\title{
Metastasis-associated protein 1 inhibits p53-induced apoptosis
}

\author{
HYO-EUN MOON, HWANJU CHEON and MYUNG-SHIK LEE \\ Department of Medicine, Samsung Medical Center, Sungkyunkwan University School of Medicine, \\ 50 Irwon-dong, Kangnam-ku, Seoul 135-710, Korea
}

Received April 24, 2007; Accepted July 3, 2007

\begin{abstract}
Metastasis-associated protein 1 (MTA1) is highly upregulated in cancer cells with metastatic potential; however, the molecular mechanism by which MTA1 increases the metastatic potential of cancer cells is far from clear. We characterized the functional consequences of MTA1 overexpression on p53-induced apoptosis of cancer cells. MTA1 was associated with p53 in a co-immunoprecipitation assay. MTA1 also had deacetylation activity on p53 in human non-small cell lung cancer cells H1299 and human hepatoma cells SK-Hep1. MTA1 attenuated the transactivation and p21 induction by p53. Moreover, MTA1 expression decreased p53mediated apoptosis. These results indicate that MTA1 inhibits p53-induced apoptosis by deacetylation of p53, which might be related to the increased metastatic potential of cancer cells with high MTA1 expression.
\end{abstract}

\section{Introduction}

MTA1 was originally isolated by differential expression screening of metastatic cell lines with high and low metastatic potentials (1). In vitro studies have shown that MTA1 increases invasiveness and migration $(2,3)$ and suggest that MTA1 may be involved in the regulation of gene expression by covalent modification of histone proteins (4). Consistent with this notion, MTA proteins are physically associated with histone deacetylases (HDAC1/2) and play a role in histone deacetylation and modulation of transcriptional activity as components of the nucleosome remodeling histone deacetylation (NuRD) complex (5). Recent reports have shown that MTA1 enhances angiogenesis by deacetylation and the

Correspondence to: Dr Myung-Shik Lee, Department of Medicine, Samsung Medical Center, Sungkyunkwan University School of Medicine, 50 Irwon-dong, Kangnam-ku, Seoul 135-710, Korea

E-mail: mslee@smc.samsung.co.kr

Abbreviations: MTA1, metastasis-associated protein 1; HDAC, histone deacetylase; NuRD, nucleosome remodeling histone deacetylation; $\mathrm{NaBu}$, sodium butyrate

Key words: metastasis-associated protein 1, p53, apoptosis, deacetylation stabilization of HIF-1 $\alpha(6,7)$, providing an important clue in understanding the mechanism of the increased metastatic potential of the cancer cells with high MTA1 expression.

Because MTA1 has deacetylating activity, MTA1 may affect the function of other proteins related to cancer progression whose activity is determined by acetylation status. In fact, MTA2, a homologue of MTA1, has been reported to modulate acetylation status and transcriptional activity of p53, while MTA2 has not been linked to metastasis or cancer progression in contrast to MTA1 (8). In response to DNA damage and other types of stress, p53 stabilizes and induces apoptosis, cell-cycle arrest, senescence and differentiation $(9,10)$. Overexpression of p53 activates a number of target genes involved in those processes such as p21CIP1/WAF1, MDM2, GADD45, Cyclin G, Noxa, Bax and PUMA (11-16) through binding to p53 consensus sequences. Therefore, the investigations of the effects of MTA1 on p53 may provide an important clue in understanding the mechanism of the increased metastasis of cancer cells with high MTA1 expression. In this study, we found that MTA1 decreases the transcriptional activity of p53 and inhibits apoptosis through deacetylation of p53.

\section{Materials and methods}

Reagents and antibodies. G418 was purchased from Invitrogen (Carlsbad, CA, USA). Protein G-agarose, protease inhibitor cocktail and FuGENE 6 Reagent were from Roche (Mannheim, Germany). Antibodies to p21, $\beta$-actin and acetylated p53 (Ac-p53) (Lys373, Lys382) were from Santa Cruz Biotechnology (Santa Cruz, CA, USA) and Upstate Biotechnology (New York, NY, USA), respectively. Antibodies to FLAG and p53 were obtained from Sigma (St. Louis, MO, USA) and Labvision (Fremont, CA, USA), respectively.

Cell culture and transfection. H1299 cells and SK-Hep1 cells were maintained in DMEM supplemented with $10 \%$ fetal bovine serum at $37^{\circ} \mathrm{C}$ in a $5 \% \mathrm{CO}_{2}$ standard incubator. To make stable MTA1 and mock transfectants, H1299 and SKHep1 cells were transfected with pcDNA3.1-human MTA1FLAG or empty pcDNA3.1 and selected with 500-600 $\mu \mathrm{g} / \mathrm{ml}$ G418 sulfate (6).

Reporter assay. HEK293 cells were transfected with pG13Luc reporter together with MTA1-FLAG using FuGENE 6 reagent. After transfection, cell lysate was assayed for luciferase 
activity using the Dual-Luciferase ${ }^{\circledR}$ Reporter assay system (Promega, Madison, WI, USA) and a luminometer (Turner Designs, Sunnyvale, CA, USA). The relative luciferase activities were calculated as previously described (17).

Western blotting. Harvested cells were lysed in a buffer (40 mM Tris-HCl, pH 7.4, 10 mM EDTA, 0.1\% NP-40, 1 mM DTT and $120 \mathrm{mM} \mathrm{NaCl}$ ) and protein concentration was measured by Bradford assay. Whole cell lysates were resolved in SDS-PAGE gels and Western blotting was performed using appropriate primary antibodies as reported (18). Protein bands were visualized with the ECL system (Amersham, Piscataway, NJ, USA).

Co-immunoprecipitation assay. After transient transfection of the target cells with His-p53, immunoprecipitation using antiFLAG or -p53 antibody, SDS-PAGE and Western blotting was performed as previously described (6).

Measurement of cell death. Adenovirus expressing p53 (Avpp53) was propagated in HEK 293 cells and concentrated by the standard $\mathrm{CsCl}$ ultracentrifugation method as previously reported (19). Cells were seeded on 96-well plates and infected with Avpp53 at a multiplicity of infection of 50 in a serumfree medium for $1 \mathrm{~h}$. To measure cell death, trypan blue exclusion test was performed by incubating cells in $0.02 \%$ trypan blue solution for $5 \mathrm{~min}$.

\section{Results}

MTA1 is associated with p53. While others and our group have reported that MTA1 stabilizes HIF-1 $\alpha$ and increases angiogenesis $(6,7)$, we hypothesized that MTA1 may additionally promote metastatic potential of cancer cells by modulating cell death. Particularly, we were interested in p53-induced apoptosis of cancer cells because p53 activity is modulated by acetylation status and MTA1 acts by deacetylating transcriptional factors such as HIF-1 $\alpha(6,7)$. To investigate the mechanism by which MTA1 affects p53induced apoptosis, we studied possible physical interaction between p53 and MTA1 using co-immunoprecipitation assay. When stable H1299 MTA1 transfectants (6) were transiently transfected with a p53 expression vector (His-p53) and immunopreciptation was performed using FLAG antibody, p53 protein was detected in the immunoprecipitates, suggesting physical association between MTA1 and 553 in vivo (Fig. 1A). Next we studied whether such physical association between MTA1 and p53 is specific to a single cell line or could be generalized to diverse cell lines. When SK-Hep1 hepatoma cells were employed instead of H1299 cells, essentially the same result was obtained, suggesting that this physical association between MTA1 and p53 is not restricted to a single cell line (Fig. 1B). These results indicate that MTA1 interacts with $\mathrm{p} 53$ in vivo, consistent with a previous study showing MTA1 binding to p53 in an in vitro GST-pull down assay (4).

MTA1 has deacetylation activity on p53. Next we studied whether MTA1 can modulate the acetylation status of p53. Western blotting using an antibody specific for acetylated p53
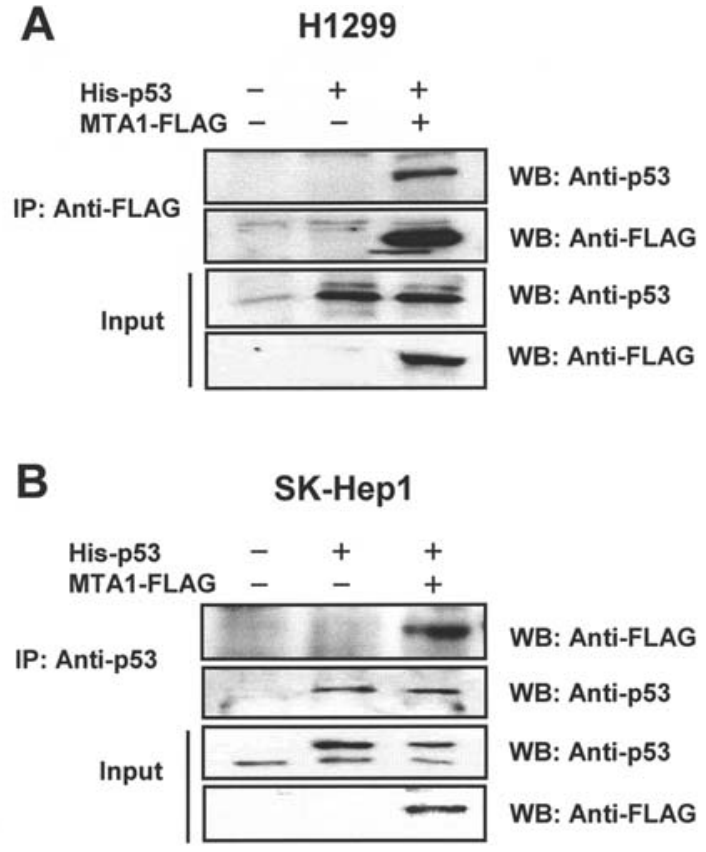

Figure 1. Association of MTA1 with p53. (A) Stable H1299 MTA1 transfectants and mock transfectants were transiently transfected with a His-p53 eukaryotic expression vector. Total cell lysate was immunoprecipitated with anti-FLAG antibody. The immunoprecipitates were analyzed by Western blotting using anti-p53 and -FLAG antibodies to study the presence of His-p53 and MTA1-FLAG. Immunoprecipitation (IP); Western blotting (WB). (B) Stable SK-Hep1 MTA1 transfectants and mock transfectants were transiently transfected with a His-p53 eukaryotic expression vector as in (A). Total cell lysate was immunoprecipitated with anti-p53 antibody. The immunoprecipitates were analyzed by Western blotting using anti-FLAG or -p53 antibodies to study the presence of His-p53, endogenous p53 and MTA1-FLAG.

A

H1299

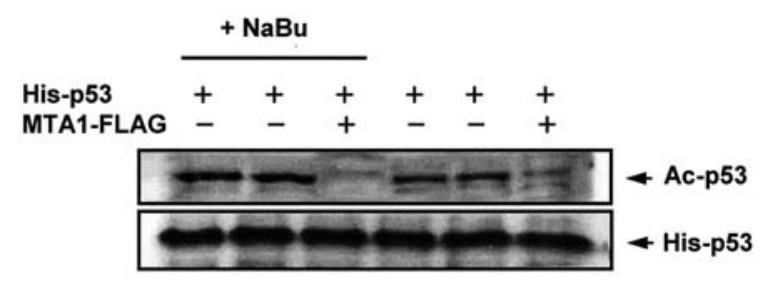

B SK-Hep1

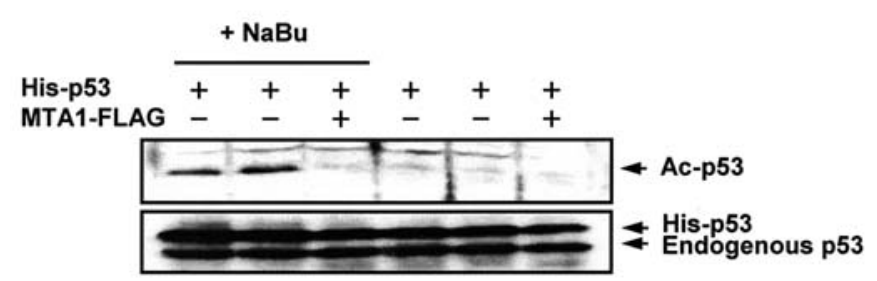

Figure 2. Deacetylation of p53 by MTA1. (A) Stable H1299 MTA1 transfectants and mock transfectants were transiently transfected with a Hisp53 eukaryotic expression vector. The expression of Ac-p53 and His-p53 were analyzed by Western blotting using anti-Ac-p53 and -p53 antibody, respectively with or without $10 \mathrm{mM} \mathrm{NaBu}$ pretreatment for $4 \mathrm{~h}$. (B) Stable SK-Hep1 MTA1 transfectants and mock transfectants were transiently transfected with a His-p53 eukaryotic expression vector. The expression of Ac-p53, His-p53 and endogenous p53 were analyzed as in (A). 
A

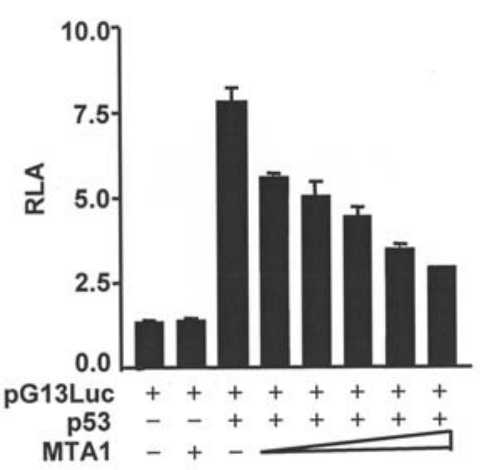

B

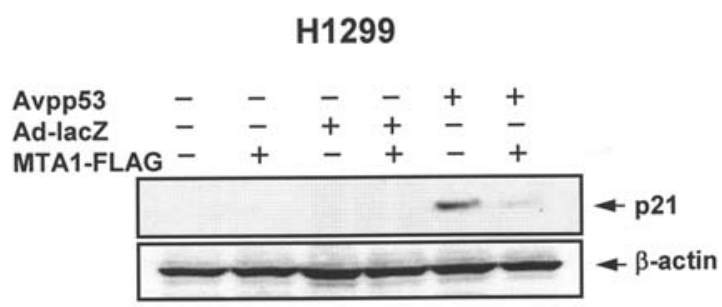

Figure 3. Decrease of p53 transcriptional activation and p21 expression by MTA1. (A) HEK293 cells were transiently transfected with $5 \mu \mathrm{g}$ pG13Luc and $0.1 \mu \mathrm{g}$ His-p53 together with varying doses of MTA1-FLAG or empty vector. After transient transfection for $24 \mathrm{~h}$, relative luciferase activity (RLA) was measured as an index of reporter activity. (B) Stable H1299 MTA1 transfectants and its respective mock transfectants were infected with an adenovirus expressing p53 (Avpp53) or a control adenovirus (Ad-lacZ). The expression of $\mathrm{p} 21$ and $\beta$-actin were analyzed by Western blotting using anti-p21 and B-actin antibody, respectively.

(Lys373, Lys382) showed that transfection of p53-null H1299 cells with His-p53 alone resulted in the expression of acetylated p53, which was increased by sodium butyrate $(\mathrm{NaBu})$, a histone deacetylase inhibitor. Stably transfected MTA1 diminished the expression of acetylated p53 both in the absence or presence of $\mathrm{NaBu}$, suggesting that MTA1 deacetylates p53 (Fig. 2A). We next conducted the same experiment using SK-Hep1 human hepatoma cells, which express wild-type p53 (20). In SK-Hep1 cells, transfection of His-p53 alone did not induce detectable acetylated p53. However, expression of acetylated p53 was detected when the cells were transfected in the presence of $\mathrm{NaBu}$. The expression of acetylated p53 detected in the presence of $\mathrm{NaBu}$ was abrogated in stable SK-Hep1 MTA1 transfectants, supporting that MTA1 has deacetylation activity on p53 (Fig. 2B).

MTA1 decreases transcriptional activation and p 21 expression by $p 53$. Next, we studied whether MTA1 modulates the transcriptional activity of p53. Reporter assay showed that cotransfection of MTA1 inhibited p53-induced transcriptional activity in a dose-dependent manner (Fig. 3A). Then, we studied the expression of p21 since p21 is one of the authentic target genes induced by p53 (11). Infection of an adenovirus expressing p53 (Avpp53) induced strong expression of p21 in H1299 cells as expected. Induction of p21 by adenoviral expression of p53 was markedly decreased in stable H1299 MTA1 transfectants compared to mock transfectants (Fig. 3B), substantiating that MTA1 decreases the induction of p21 probably through attenuation of transactivation by $\mathrm{p} 53$.
A

H1299

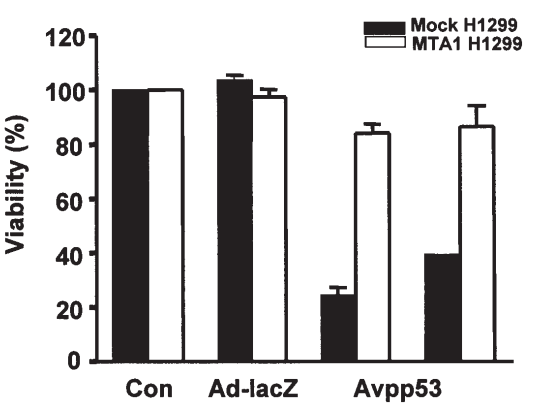

B

SK-Hep1

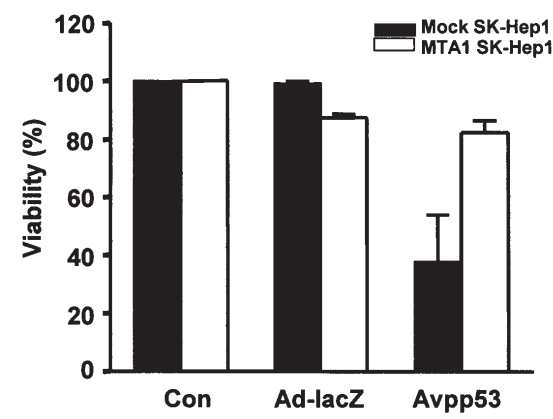

Figure 4. Inhibition of p53-induced apoptosis by MTA1. (A) Stable H1299 MTA1 transfectants (open bars) and mock transfectants (filled bars) were seeded on 96-well plates and infected with adenovirus expressing p53 (Avpp53) or control Ad-lacZ. After incubation for 24 to $36 \mathrm{~h}$, trypan blue exclusion test was performed by incubating cells in $0.02 \%$ trypan blue solution for $5 \mathrm{~min}$. (B) Stable SK-Hep1 MTA1 transfectants (open bars) and mock transfectants (filled bars) were seeded on 96-well plates. Infection with adenovirus expressing p53 (Avpp53) and trypan blue exclusion test were conducted as in $(\mathrm{A})$.

MTA1 inhibits apoptosis induced by p53. Next, we explored the biological consequence of MTA1-mediated modulation of p53 by studying the effect of MTA1 on p53-induced cell death. Trypan blue exclusion assay demonstrated that cell death by adenoviral expression of p53 was attenuated significantly in both H1299 and SK-Hep1 MTA1 transfectants, compared to their respective mock transfectants (Fig. 4A and B), suggesting that MTA1 inhibits apoptosis induced by p53 in multiple cell lines by deacetylating p53 and attenuating transactivation by $\mathrm{p} 53$.

\section{Discussion}

To understand the mechanism by which MTA1 increases the metastatic potential of cancer cells, we studied the functional consequences of MTA1 overexpression on p53. First, we confirmed physical association between p53 and MTA1 by using co-immunoprecipitation assay. We then showed that MTA1 deacetylates p53. In one cell line (H1299), acetylated p53 was detected even in the absence of $\mathrm{NaBu}$, which was decreased by MTA1, strongly supporting that MTA1 deacetylates p53 in vivo. We observed that the effect of MTA1 was not significantly affected by $\mathrm{NaBu}$. The effect of $\mathrm{NaBu}$ as an inhibitor of deacetylation may vary according to the target proteins and the effect of $\mathrm{NaBu}$ on MTA1 could be different from that on other major deacetylators such as HDACs $(7,8)$. 
Probably because of deacetylation activity on p53, MTA1 decreased transactivation by $\mathrm{p} 53$ and led to the abrogation of the induction of p21, a classical p53 target protein. Probably through the abrogation of p53 target gene expression, MTA1 transfection inhibited p53-induced apoptosis. While MTA2 has been reported to have deacetylation activity on p53 (8), the effect of MTA1 on p53-induced apoptosis has not been reported to our knowledge. Our data may provide a novel clinical implication related to metastasis because MTA2, despite its deacetylation activity on $\mathrm{p} 53$, has not been shown to be involved in metastasis or carcinogenesis in contrast to MTA1 (4). In conclusion, MTA1 could be a member of p53 deacetylators such as PML, Ras, SIRT1 (21) that are important modulators of p53 activity (22). While p53 deacetylation alone cannot explain high metastatic potential of cancer cells with MTA1 overexpression, MTA1 may be able to promote metastasis of cancer cells by inhibiting p53-induced cell death described in this report and also by deacetylating HIF- $1 \alpha$ and increasing its stability as previously reported $(6,7)$.

We have observed that MTA1 was overexpressed in mitochondrial DNA-depleted $\rho^{0}$ cells (19, and unpublished data). Considering frequent mitochondrial mutations in malignant cells (23), MTA1 overexpression might be related to the increased malignant potential of cancer cells with mitochondrial dysfunction/mutations (23) and increased resistance of cells with mitochondrial dysfunction against cell death $(19,24)$.

Collectively, these results indicate that MTA1 inhibits p53-dependent apoptosis by deacetylating p53 and attenuating transactivation by $\mathrm{p} 53$, which may contribute to the enhanced metastatic potential of cancer cells with high MTA1 expression. Because MTA1 modulates both p53 and HIF-1 $\alpha$, the two important molecules in carcinogenesis and metastasis, MTA1 could be a molecular target for inhibition of cancer progression and metastasis, which are important clinical points in determining the final outcome of the disease in patients with advanced cancers.

\section{Acknowledgements}

This study was supported by the Nano/Bio Science Program Grant (2004-00716) and 21C Frontier Functional Proteomics Project (FPR05C1-160). M.-S.L. is an awardee of the SRC Grants/R01-2005-000-10326-0 from the Korea Science \& Engineering Foundation. We thank Professor Paul A. Wade (Department of Pathology and Laboratory Medicine, Emory University School of Medicine, USA) for his kind provision of human MTA1-FLAG eukaryotic expression vector, Dr Kyoung-Sook Park (Molecular Therapy Research Center, Sungkyunkwan University, Samsung Medical Center) for pG13Luc luciferase reporter gene and Dr Seung-Hoon Lee (Molecular Therapy Research Center, Sungkyunkwan University, Samsung Medical Center) for His-p53 eukaryotic expression vector.

\section{References}

1. Toh Y, Pencil SD and Nicolson GL: A novel candidate metastasis-associated gene, mta1, differentially expressed in highly metastatic mammary adenocarcinoma cell lines. cDNA cloning, expression and protein analyses. J Biol Chem 269: 22958-22963, 1994.

2. Oki E, Oda S, Tokunaga E, et al: Overexpression of the MTA1 gene in gastrointestinal carcinomas: correlation with invasion and metastasis. Int J Cancer 74: 459-463, 1997.

3. Mahoney MG, Simpson A, Jost M, et al: Metastasis-associated protein (MTA)1 enhances migration, invasion and anchorageindependent survival of immortalized human keratinocytes. Oncogene 21: 2161-2170, 2002.

4. Yao YL and Yang WM: The metastasis-associated proteins 1 and 2 form distinct protein complexes with histone deacetylase activity. J Biol Chem 278: 42560-42568, 2003.

5. Xue Y, Wong J, Moreno GT, et al: NURD, a novel complex with both ATP-dependent chromatin-remodeling and histone deacetylase activities. Mol Cell 2: 851-861, 1998 .

6. Moon HE, Cheon H, Chun KH, et al: Metastasis-associated protein 1 enhances angiogenesis by stabilization of HIF-1 $\alpha$. Oncol Rep 16: 929-935, 2006.

7. Yoo YG, Kong G and Lee MO: Metastasis-associated protein 1 enhances stability of hypoxia-inducible factor-1alpha protein by recruiting histone deacetylase 1. EMBO J 25: 1231-1241, 2006.

8. Luo J, Su F, Chen D, et al: Deacetylation of p53 modulates its effect on cell growth and apoptosis. Nature 408: 377-381, 2000.

9. Levine AJ: p53, the cellular gatekeeper for growth and division. Cell 88: 323-331, 1997.

10. Vogelstein B, Lane D and Levine AJ: Surfing the p53 network. Nature 408: 307-310, 2000

11. El-Deiry WS, Tokino T, Velculescu VE, et al: a potential mediator of p53 tumor suppression. Cell 75: 817-825, 1993.

12. Barak Y, Juven T, Haffner R and Oren M: Mdm2 expression is induced by wild type p53 activity. EMBO J 12: 461-468, 1993.

13. Kastan MB, Zhan Q, el-Deiry WS, et al: A mammalian cell cycle checkpoint pathway utilizing p53 and GADD45 is defective in ataxia-telangiectasia. Cell 71: 587-597, 1992.

14. Okamoto $\mathrm{K}$ and Beach D: Cyclin $\mathrm{G}$ is a transcriptional target of the p53 tumor suppressor protein. EMBO J 13: 4816-4822, 1994.

15. Miyashita T, Kitada S, Krajewski S, et al: Overexpression of the Bcl-2 protein increases the half-life of p21Bax. J Biol Chem 270: 26049-26052, 1995.

16. Chipuk JE, Bouchier-Hayes L, Kuwana T, et al: PUMA couples the nuclear and cytoplasmic proapoptotic function of p53. Science 309: 1732-1735, 2005.

17. Kern SE, Pietenpol JA, Thiagalingam S, et al: Oncogenic forms of p53 inhibit p53-regulated gene expression. Science 256: 827-830, 1992.

18. Chang I, Kim S, Kim JY, et al: Nuclear factor kappaB protects pancreatic beta-cells from tumor necrosis factor-alpha-mediated apoptosis. Diabetes 52: 1169-1175, 2003.

19. Park SY, Chang I, Kim JY, et al: Resistance of mitochondrial DNA-depleted cells against cell death: role of mitochondrial superoxide dismutase. J Biol Chem 279: 7512-7520, 2004.

20. Schumacher G, Scheunert S, Rueggeberg A, et al: A very low toxic agent induces apoptosis and reduced growth of human hepatocellular carcinoma cells. J Gastroenterol Hepatol 21: 1207-1212, 2006

21. Vaziri H, Dessain SK, Ng Eaton E, et al: hSIR2(SIRT1) functions as an NAD-dependent p53 deacetylase. Cell 107: 149-159, 2001.

22. Prives C and Manley JL: Why is p53 acetylated? Cell 107: 815-818, 2001.

23. Simonnet H, Alazard N, Pfeiffer K, et al: Low mitochondrial respiratory chain content correlates with tumor aggressiveness in renal cell carcinoma. Carcinogenesis 23: 759-768, 2002.

24. Kim JY, Kim Y-H, Chang I, et al: Resistance of mitochondrial DNA-deficient cells to TRAIL: Role of Bax in TRAIL-induced apoptosis. Oncogene 21: 3139-3148, 2002. 